PROCEEDINGS OF THE

AMERICAN MATHEMATICAL SOCIETY

Volume 136, Number 12, December 2008, Pages 4201-4209

S 0002-9939(08)09467-7

Article electronically published on July 24, 2008

\title{
NONABELIAN THETA FUNCTIONS OF POSITIVE GENUS
}

\author{
ARZU BOYSAL
}

(Communicated by Ted Chinburg)

\begin{abstract}
Let $\mathcal{C}_{g}$ be a smooth projective irreducible curve over $\mathbb{C}$ of genus $g \geq 1$ and let $\left\{p_{1}, \ldots, p_{s}\right\}$ be a set of distinct points on $\mathcal{C}_{g}$. We fix a nonnegative integer $\ell$ and denote by $M_{g}(\underline{p}, \underline{\lambda})$ the moduli space of parabolic semistable vector bundles of rank $r$ on $\mathcal{C}_{g}$ with trivial determinant and fixed parabolic structure of type $\underline{\lambda}=\left(\lambda_{1}, \ldots, \lambda_{s}\right)$ at $\underline{p}=\left(p_{1}, \ldots, p_{s}\right)$, where each weight $\lambda_{i}$ is in $P_{\ell}(\mathrm{SL}(r))$. On $M_{g}(\underline{p}, \underline{\lambda})$ there is a canonical line bundle $\mathcal{L}(\underline{\lambda}, \ell)$, whose global sections are called generalized parabolic $\mathrm{SL}(r)$-theta functions of order $\ell$. In this paper we prove the existence of such nonzero nonabelian theta functions, thus establishing a part of higher genus generalizations of the celebrated saturation conjectures.
\end{abstract}

\section{INTRODUCTION}

Let $\mathcal{C}_{g}$ be a smooth projective irreducible curve over $\mathbb{C}$ of genus $g \geq 1$ and let $\left\{p_{1}, p_{2}, \ldots, p_{s}\right\}$ be a set of distinct points on $\mathcal{C}_{g}$.

We fix a nonnegative integer $\ell$ and denote by $M_{\mathcal{C}_{g}}^{\mathrm{SL}(r)}(\underline{p}, \underline{\lambda})$ (for short $M_{g}(\underline{p}, \underline{\lambda})$ ) the moduli space of parabolic semistable vector bundles of rank $r$ on $\mathcal{C}_{g}$, with trivial determinant and fixed parabolic structure of type $\underline{\lambda}=\left(\lambda_{1}, \ldots, \lambda_{s}\right)$ at $p=$ $\left(p_{1}, \ldots, p_{s}\right)$ (see [18] or [16] for definitions), where each weight $\lambda_{i}$ is in $P_{\ell}(\operatorname{SL}(\bar{r}))$; that is, each $\lambda_{i}$ is dominant integral and $\lambda_{i}\left(H_{\theta}\right) \leq \ell$ (cf. section 2.2).

On $M_{g}(\underline{p}, \underline{\lambda})$ there is a natural line bundle $\mathcal{L}(\underline{\lambda}, \ell)$ whose global sections can be seen as parabolic generalizations of the nonabelian version of classical theta functions on the Jacobian of $\mathcal{C}_{g}$, and are often called generalized parabolic $\operatorname{SL}(r)$ theta functions (for short nonabelian theta functions) of order $\ell$.

One of the motivations for our work comes from the Knutson-Tao saturation theorem [13] and its generalizations (see Fulton [10] for a survey). Recall that if $V_{\lambda_{1}}, \ldots, V_{\lambda_{s}}$ are irreducible representations of $\operatorname{SL}(r)$ such that $\sum_{1}^{s} \lambda_{i}$ lies in the root lattice (for simplicity also assume that each $\lambda_{i}$ is strictly dominant), then one can interpret $\left(V_{\lambda_{1}} \otimes \cdots \otimes V_{\lambda_{s}}\right)^{\mathrm{SL}(r)}$ as $H^{0}\left((\mathrm{SL}(r) / B)^{s} / / \mathrm{SL}(r), L\right)$, where $L$ is the descent via GIT of the line bundle $\mathbb{C}_{-\lambda_{1}} \otimes \cdots \otimes \mathbb{C}_{-\lambda_{s}}$ to the GIT quotient $(\mathrm{SL}(r) / B)^{s} / / \mathrm{SL}(r)$. Let $M$ be the GIT quotient. According to the Knutson-Tao saturation theorem, for any positive integer $N$,

$$
H^{0}\left(M, L^{N}\right) \neq 0 \text { if and only if } H^{0}(M, L) \neq 0 .
$$

Received by the editors August 22, 2007, and, in revised form, November 16, 2007.

2000 Mathematics Subject Classification. Primary 14H60; Secondary 22E65.

Key words and phrases. Theta functions, parabolic vector bundles, factorization rules, fusion product, PRV, saturation conjecture.

(C)2008 American Mathematical Society Reverts to public domain 28 years from publication 
Now $L$ is ample on $M$, so the above statement can be rewritten as

$$
M \neq \emptyset \text { if and only if } H^{0}(M, L) \neq 0 .
$$

One can view $M$ as a 'classical limit' of the moduli of parabolic bundles (actually the limiting case for large level, $g=0)$ and $H^{0}(M, L)$ as the classical limit of nonabelian theta functions. Therefore the Knutson-Tao theorem leads to the expectation that whenever moduli spaces $M$ (of type A) are nonempty, nonzero sections of suitable line bundles on them exist. For $g=0$ this is established by works of Belkale on quantum horn and saturation conjectures $[3$. We will verify this saturation principle in higher genus. Here $M$ is the moduli of parabolic bundles $M_{g}(\underline{p}, \underline{\lambda}$ ), which is never empty for $g \geq 1$ (cf. section 2.4), so the expectation is that nonzero theta functions should exist, which we prove. Our paper should therefore be seen as a higher genus saturation theorem. In fact, we give the following nontrivial lower bound for their rank:

Theorem 1.1. Let $\mathcal{C}_{g}$ be a smooth projective irreducible curve over $\mathbb{C}$ of genus $g \geq 1$ and let $\left\{p_{1}, p_{2}, \ldots, p_{s}\right\}$ be a set of distinct points on $\mathcal{C}_{g}$. Fix a nonnegative integer $\ell$, and let $M_{g}(\underline{p}, \underline{\lambda})$ be the moduli space of parabolic semistable $\mathrm{SL}(r)$-bundles of type $\underline{\lambda}=\left(\lambda_{1}, \lambda_{2}, \ldots, \lambda_{s}\right)$ at $\underline{p}=\left(p_{1}, p_{2}, \ldots, p_{s}\right)$ with each $\lambda_{i} \in P_{\ell}(\operatorname{SL}(r))$.

If $\sum_{1}^{s} \lambda_{i}$ lies in the root lattice of $\mathrm{SL}(r)$, then

$$
\operatorname{dim} H^{0}\left(M_{g}(\underline{p}, \underline{\lambda}), \mathcal{L}(\underline{\lambda}, \ell)\right) \geq\left(\sharp P_{\ell}(\mathrm{SL}(r))\right)^{g-1},
$$

where $\sharp P_{\ell}(\mathrm{SL}(r))=(\ell+r-1) ! /(\ell !(r-1)$ !).

In the proof of this main result we make essential use of the canonical identification of sections of $\mathcal{L}(\underline{\lambda}, \ell)$ with the space of conformal blocks (see Sorger [20] for a survey).

The outline of the proof of the above main theorem is as follows: under the above-mentioned correspondence, the dimension of $H^{0}\left(M_{g}(\underline{p}, \underline{\lambda}), \mathcal{L}(\underline{\lambda}, \ell)\right)$ obeys the 'factorization rule' and has the 'propagation of vacua' property as given by 22 (cf. Proposition 3.2). Using these, we reduce the problem of determining nontrivial lower bounds for the dimension of sections of $\mathcal{L}(\underline{\lambda}, \ell)$ to the case of genus $g=1$ (cf. Lemma 4.1).

For $g=1$, we first prove the existence of a nonzero section for the particular case of one marking (i.e. $s=1$ ) using a Parthasarathy-Ranga Rao-Varadarajan (PRV) type result for the fusion product of $\mathrm{SL}(r)$ representations given by Belkale. We show that for any $\lambda \in P_{\ell}(\operatorname{SL}(r))$ that is also in the root lattice, there exists a PRV weight $\mu \in P_{\ell}(\mathrm{SL}(r))$ such that spaces of genus zero parabolic theta functions of type $\left(\lambda, \mu, \mu^{*}\right)$ are nonzero (cf. Proposition 4.2). We then reduce the case of an arbitrary number of markings to this case (cf. Proposition 4.3). By these two propositions, we have $H^{0}\left(M_{1}(\underline{p}, \underline{\lambda}), \mathcal{L}(\underline{\lambda}, \ell)\right) \neq 0$ (which is precisely the claim in Theorem 1.1 for $g=1$ ). Then, by virtue of the above-mentioned reduction to genus 1 , Theorem 1.1 follows.

\section{Preliminaries}

We set up the notation and recall basic definitions and concepts. The main reference for sections 2.1 and 2.2 is 6 . Sections 2.3 onwards contain results on moduli spaces of parabolic bundles pertinent to this paper. 
2.1. Let $G$ be a connected, simply connected, simple affine algebraic group over $\mathbb{C}$. This will be our assumption on $G$ unless otherwise stated. We fix a Borel subgroup $B$ of $G$ and a maximal torus $T \subset B$. Let $\mathfrak{h}, \mathfrak{b}$ and $\mathfrak{g}$ denote the Lie algebra of $T$, $B$ and $G$ respectively. Let $R=R(\mathfrak{h}, \mathfrak{g}) \subset \mathfrak{h}^{*}$ be the root system; there is the root space decomposition $\mathfrak{g}=\mathfrak{h} \oplus\left(\bigoplus_{\alpha \in R} \mathfrak{g}_{\alpha}\right)$. We fix a basis $\Delta=\left\{\alpha_{1}, \ldots, \alpha_{r}\right\}$ of $R$, where $r$ is the rank of $G$. Let $\mathfrak{h}_{\mathbb{R}}$ denote the real span of elements of $\mathfrak{h}$ dual to $\Delta$. For each root $\alpha$, denote by $H_{\alpha}$ the unique element of $\left[\mathfrak{g}_{\alpha}, \mathfrak{g}_{-\alpha}\right]$ such that $\alpha\left(H_{\alpha}\right)=2$; we denote by $\breve{Q}$ the lattice spanned by $H_{\alpha}$. We also choose elements $E_{\alpha} \in \mathfrak{g}_{\alpha}$ and $F_{\alpha} \in \mathfrak{g}_{-\alpha}$ that span, together with $H_{\alpha}$, a Lie subalgebra of $\mathfrak{g}$ isomorphic to $\mathfrak{s l}_{2}$. Let $\left\{\omega_{i}\right\}_{1 \leq i \leq r}$ be the set of fundamental weights, defined as the basis of $\mathfrak{h}^{*}$ dual to $\left\{H_{\alpha_{i}}\right\}_{1 \leq i \leq r}$. Define the weight lattice $P=\left\{\lambda \in \mathfrak{h}^{*}: \lambda\left(H_{\alpha}\right) \in \mathbb{Z}, \forall \alpha \in R\right\}$; we denote the set of dominant weights by $P^{+}$.

Let $(\mid)$ denote the Killing form on $\mathfrak{g}$ normalized such that $\left(H_{\theta} \mid H_{\theta}\right)=2$, where $\theta$ is the highest root of $G$. We will use the same notation for the restricted form on $\mathfrak{h}$ and the induced form on $\mathfrak{h}^{*}$. We introduce the Weyl group $W:=N_{G}(T) / T$. The Killing form is positive definite on $\mathfrak{h}_{\mathbb{R}}$, thus induces the following canonical isomorphism:

$$
F: \mathfrak{h}_{\mathbb{R}}^{*} \rightarrow \mathfrak{h}_{\mathbb{R}} ; \quad \alpha \mapsto\left(2 /\left(H_{\alpha} \mid H_{\alpha}\right)\right) H_{\alpha}
$$

with $F^{-1}\left(H_{\alpha}\right)=(2 /(\alpha \mid \alpha)) \alpha$. Under the above identification, $W$ is generated by elements $w_{\alpha}$ for $\alpha \in \Delta$ acting on $\mathfrak{h}_{\mathbb{R}}^{*}$ as $w_{\alpha} \beta=\beta-\beta\left(H_{\alpha}\right) \alpha$. Thus, given a weight $\beta \in P$ that is also in the root lattice, $w \beta-\beta$ is again in the root lattice for any $w \in W$. Let $w_{0}$ denote the longest element in the Weyl group. Then for any $\lambda \in P$, the dual of $\lambda$ denoted by $\lambda^{*}$ is $-w_{0} \lambda$.

2.2. For a positive integer $\ell$, define the set $P_{\ell}(G):=\left\{\lambda \in P^{+}: \lambda\left(H_{\theta}\right) \leq \ell\right\}$; it is finite and stable under taking the dual. In particular, for $G=\mathrm{SL}(r)$ and $\lambda=\sum_{i=1}^{r-1} a_{i} \omega_{i}, P_{\ell}(\mathrm{SL}(r))=\left\{a_{i} \in \mathbb{Z}_{\geq 0}: \sum_{i=1}^{r-1} a_{i} \leq \ell\right\}$.

Let $\mathfrak{A}=\left\{h \in \mathfrak{h}_{+}: \theta(h) \leq 1\right\}$ denote the (closed) fundamental alcove. $\mathfrak{A}$ is a fundamental domain for the action on $\mathfrak{h}_{\mathbb{R}}$ of the affine Weyl group $W_{\text {aff }}$, which is the semidirect product of $W$ and $\check{Q}$. By virtue of the normalization in the Killing form, for any $\lambda \in P_{\ell}(G)$,

$$
\theta(F(\lambda))=\lambda(F(\theta))=\lambda\left(H_{\theta}\right) .
$$

Thus, $\lambda / \ell$ determines a unique element $F(\lambda / \ell)$ in $\mathfrak{A}$. Often we shall implicitly identify a weight in $P_{\ell}(G)$ with the corresponding element in $\mathfrak{A}$ and hence with an orbit of $\mathfrak{h}_{\mathbb{R}} / W_{\text {aff }}$.

2.3. Let $\mathcal{C}_{g}$ be a smooth projective irreducible curve over $\mathbb{C}$ of genus $g \geq 1$ and let $\underline{p}=\left(p_{i}\right)_{1 \leq i \leq s}$ be distinct points on $\mathcal{C}_{g}$ labeled by weights $\underline{\lambda}=\left(\lambda_{i}\right)_{1 \leq i \leq s}$, with each $\bar{\lambda}_{i}$ in $P_{\ell}(G)$. Then one can construct (see [16]) the (algebraic) moduli stack of quasiparabolic $G$-bundles associated to this data, denoted by $\mathcal{M}_{g}^{G}(\underline{p}, \underline{\lambda})$. There exists a (coarse) moduli space for $\mathcal{M}_{g}^{G}(p, \underline{\lambda})$ which is a projective variety and its points are equivalence classes of parabolic semistable $G$-bundles of fixed topological type and fixed parabolic structure ([5], Theorem II). We denote this space by $M_{g}^{G}(\underline{p}, \underline{\lambda})$.

2.4. We recall that the real analytic space underlying $M_{g}^{G}(\underline{p}, \underline{\lambda})$ admits a description as the space of representations of the fundamental group $\pi_{1}\left(C_{g} \backslash\left\{p_{1}, \ldots, p_{s}\right\}, b\right)$ into a fixed compact form $K$ of $G$, up to conjugation ([5, Proposition 2.3, or 
21] for $G=\mathrm{SL}(r)$ see [18, Theorem 4.1). As is well known, the set of conjugacy classes of $K$ is in bijective correspondence with $\mathfrak{h}_{\mathbb{R}} / W_{\text {aff }}$. For a collection of weights $\underline{\lambda}=\left(\lambda_{1}, \ldots, \lambda_{s}\right)$ in $P_{\ell}(G)$, we denote by $C_{i}$ the conjugacy class of $\exp \left(2 \pi \sqrt{-1} F\left(\lambda_{i} / \ell\right)\right)$ for $1 \leq i \leq s$. Then we have the following well known isomorphism as real analytic spaces:

$$
\begin{aligned}
M_{g}^{G}(\underline{p}, \underline{\lambda}) \simeq\left\{\left(k_{1}, \ldots, k_{2 g}, h_{1}, \ldots, h_{s}\right) \in K^{2 g+s}:\right. \\
\\
\left.\prod_{i=1}^{g}\left[k_{i}, k_{i+g}\right]=\prod_{j=1}^{s} h_{j}, \quad h_{i} \in C_{i}\right\} / \operatorname{Ad} K,
\end{aligned}
$$

where / Ad $K$ refers to the quotient under the diagonal adjoint action of $K$ on $K^{2 g+s}$. For $g \geq 1$, under the above identification $(*)$ and using a theorem of Gotô 11, which states that the image of the commutator map $K \times K \rightarrow K ;\left(k_{1}, k_{2}\right) \mapsto\left[k_{1}, k_{2}\right]$ is surjective, the moduli space $M_{g}^{G}(\underline{p}, \underline{\lambda})$ is nonempty for any collection of weights $\underline{\lambda}$ in $P_{\ell}(G)$.

2.5. There is a natural line bundle $\mathcal{L}(\underline{\lambda}, \ell)$ on the moduli stack (see [16], section 8.9, for the construction). It is known that for $G=\mathrm{SL}(r), \mathcal{L}(\underline{\lambda}, \ell)$ descends to the moduli space $M_{g}(\underline{p}, \underline{\lambda})([7],[19])$. Moreover,

$$
H^{0}\left(\mathcal{M}_{g}^{\mathrm{SL}(r)}(\underline{p}, \underline{\lambda}), \mathcal{L}(\underline{\lambda}, \ell)\right) \simeq H^{0}\left(M_{g}^{\mathrm{SL}(r)}(\underline{p}, \underline{\lambda}), \mathcal{L}(\underline{\lambda}, \ell)\right) .
$$

For proof of the above canonical identification of sections we refer to [19, Proposition 5.2; the nonparabolic case is proved in [2, Propositions 8.3 and 8.4.

We will make essential use of the following canonical identification:

$$
H^{0}\left(\mathcal{M}_{g}^{G}(\underline{p}, \underline{\lambda}), \mathcal{L}(\underline{\lambda}, \ell)\right) \simeq V_{\mathcal{C}_{g}}^{G}(\underline{p}, \underline{\lambda}),
$$

where the space on the right-hand side is called the space of conformal blocks and will be defined in the next section. The above isomorphism is proved for the nonparabolic case independently by Beauville-Laszlo 2, Faltings 8 and KumarNarasimhan-Ramanathan [15]; parabolic variants are given by Pauly [19] and Laszlo-Sorger [16.

Finally, by the above list of identifications, we have

$$
H^{0}\left(M_{g}^{\mathrm{SL}(r)}(\underline{p}, \underline{\lambda}), \mathcal{L}(\underline{\lambda}, \ell)\right) \simeq V_{\mathcal{C}_{g}}^{\mathrm{SL}(r)}(\underline{p}, \underline{\lambda}) .
$$

\section{Conformal Blocks, their Degeneration properties, FUSION PRODUCT AND PRV COMPONENTS}

We recall the definition of the space of conformal blocks as given by TsuchiyaUeno-Yamada 22. This space is identified with generalized parabolic $G$-theta functions of level $\ell$ as recalled in section 2.5 .

Let $\tilde{\mathfrak{g}}=\mathfrak{g} \otimes \mathbb{C}((z)) \oplus \mathbb{C C}$ denote the (untwisted) affine lie algebra associated to $\mathfrak{g}$ over $\mathbb{C}((z))$, with the lie bracket given by $[x \otimes f, y \otimes g]=[x, y] \otimes f g+((x \mid y) \operatorname{Res}(g d f))$. $\mathrm{C}$ and $[\mathfrak{g}, \mathrm{C}]=0$ for $x, y \in \mathfrak{g}$ and $f, g \in \mathbb{C}((z))$.

We fix a positive integer $\ell$, called the level. It is well known from representation theory of affine Lie algebras that for each $\lambda \in P_{\ell}(G)$ there exists a unique (up to isomorphism) left $\tilde{\mathfrak{g}}$-module $\mathcal{H}_{\lambda}$, called the integrable highest weight $\tilde{\mathfrak{g}}$-module, with the central element $\mathrm{C}$ acting as $\ell \cdot \mathrm{Id}$ (see e.g. [12]). 
Definition 3.1. The space of conformal blocks on $\mathcal{C}_{g}$ with marked points $\left(p_{1}, \ldots\right.$, $\left.p_{k}\right)$ and weights $\left(\lambda_{1}, \cdots, \lambda_{k}\right)$ attached to them (each $\left.\lambda_{i} \in P_{\ell}(G)\right)$ with central charge $\ell$ is

$$
V_{\mathcal{C}_{g}}^{G}(\underline{p}, \underline{\lambda}):=\left[\mathcal{H}_{\lambda_{1}} \otimes \mathcal{H}_{\lambda_{2}} \otimes \cdots \otimes \mathcal{H}_{\lambda_{k}}\right]_{\mathfrak{g} \otimes \mathcal{O}\left(\mathcal{C}_{g} \backslash\left\{p_{1}, \ldots, p_{k}\right\}\right)},
$$

where $\mathcal{O}\left(\mathcal{C}_{g} \backslash\left\{p_{1}, \ldots, p_{k}\right\}\right)$ denotes the ring of algebraic functions on the punctured curve $\mathcal{C}_{g} \backslash\left\{p_{1}, \ldots, p_{k}\right\}$, and the notation []$_{\mathfrak{g} \otimes \mathcal{O}\left(\mathcal{C}_{g} \backslash\left\{p_{1}, \ldots, p_{k}\right\}\right)}$ denotes taking coinvariants with respect to the Lie algebra in the subscript. (See 22] or 1] for details of the action used in taking coinvariants.)

These vector spaces form a projectively flat vector bundle over the moduli space of curves with marked points $([22],[9])$; hence the rank of the vector space $V_{\mathcal{C}_{g}}^{G}(\underline{p}, \underline{\lambda})$ depends only on the genus $g$ and on weights $\underline{\lambda}$. Denote this common rank by $n_{g}(\underline{\lambda})$, that is, $n_{g}(\underline{\lambda})=\operatorname{dim} V_{\mathcal{C}_{g}}^{G}(\underline{p}, \underline{\lambda})$. Its value is given by the Verlinde formula ([2], $\underline{8}$, [23]).

Below, we state the principle of 'propagation of vacua' and the 'factorization rule' for conformal blocks only in terms of their dimensions, as we will not need explicit isomorphisms.

Proposition 3.2. For any collection of weights $\underline{\lambda}$ in $P_{\ell}(G)$ :

(a) (Propogation of vacua, 22, Proposition 2.2.3]) $n_{g}(\underline{\lambda}, 0)=n_{g}(\underline{\lambda})$.

(b) (Factorization rule, [22, Proposition 2.2.6])

$$
n_{g}(\underline{\lambda})=\sum_{\nu \in P_{\ell}(G)} n_{g-1}\left(\underline{\lambda}, \nu, \nu^{*}\right) .
$$

Let $\mathcal{R}(\mathfrak{g})$ denote the Grothendieck ring of finite dimensional representations of $\mathfrak{g}$. There is a bijection of $P^{+}$onto $\mathcal{R}(\mathfrak{g})$ where a dominant weight $\lambda$ is associated to the isomorphism class of the simple $\mathfrak{g}$-module $V_{\lambda}$ containing a highest weight vector with weight $\lambda$. The multiplicative structure in $\mathcal{R}(\mathfrak{g})$ is induced from the tensor product of two representations.

The fusion ring associated to $\mathfrak{g}$ and the nonnegative integer $\ell, \mathcal{R}_{\ell}(\mathfrak{g})$, is a free $\mathbb{Z}$-module with basis $\left\{V_{\lambda}, \lambda \in P_{\ell}(G)\right\}$. The ring structure is defined as

$$
V_{\lambda} \otimes^{F} V_{\mu}:=\bigoplus_{\nu \in P_{\ell}(G)} n_{\lambda, \mu}\left(\nu^{*}\right) V_{\nu},
$$

where $n_{\lambda, \mu}\left(\nu^{*}\right):=\operatorname{dim}\left[\mathcal{H}_{\lambda} \otimes \mathcal{H}_{\mu} \otimes \mathcal{H}_{\nu}^{*}\right]_{\mathbf{g} \otimes \mathcal{O}\left(\mathbb{P}^{1}-\{3 \text { points }\}\right)}=n_{0}\left(\lambda, \mu, \nu^{*}\right)$. The product $\otimes^{F}$ is clearly commutative; it is also associative by virtue of the 'factorization rule'.

For completeness, we will outline the necessity of the assumption ' $\sum_{1}^{s} \lambda_{i}$ lies in the root lattice of $\mathrm{SL}(r)^{\prime}$ in Theorem 1.1 $V_{\mathbb{P}^{1}}^{G}(\underline{p}, \underline{\lambda})$ is isomorphic to the largest quotient of $V_{\lambda_{1}} \otimes \cdots \otimes V_{\lambda_{s}}$ on which $\mathfrak{g}$ and $\left(E_{\theta} \otimes z^{-1}\right)^{\ell+1}$ act trivially (see the Appendix in [8], or [1]). Therefore $V_{\mathbb{P}^{1}}^{G}(\underline{p}, \underline{\lambda})$ is empty if $\sum_{1}^{s} \lambda_{i}$ fails to lie in the root lattice. Then, inductively by Proposition $\underline{3.2}(b), V_{\mathcal{C}_{g}}^{G}(\underline{p}, \underline{\lambda})$ is also empty for any $g \geq 1$.

We now recall (a version of) the Parthasarathy-Ranga Rao-Varadarajan (PRV) conjecture (already proven) for the tensor product of two simple $\mathfrak{g}$-modules. Let $V_{\lambda_{1}}$ and $V_{\lambda_{2}}$ be two finite dimensional irreducible $\mathfrak{g}$-modules with highest weights $\lambda_{1}$ and $\lambda_{2}$ respectively. Then for any $w \in W$ the irreducible $\mathfrak{g}$-module $V_{\overline{\lambda_{1}+w \lambda_{2}}}$ occurs with multiplicity at least one in $V_{\lambda_{1}} \otimes V_{\lambda_{2}}$, where $\overline{\lambda_{1}+w \lambda_{2}}$ denotes the unique dominant element in the $W$-orbit of $\lambda_{1}+w \lambda_{2}$ ([14, [17]). Weights of this 
form are called PRV-weights. An analogous theorem for the fusion product of $\mathfrak{s l}(r)$ representations is given by Belkale. Before stating this theorem we observe the following.

Given any $\lambda_{1}, \lambda_{2} \in P_{\ell}(G)$ and $w \in W_{\text {aff }}$, there exists $\tilde{w} \in W_{\text {aff }}$ such that $\tilde{w}\left(F\left(\lambda_{1} / \ell\right)+w F\left(\lambda_{2} / \ell\right)\right) \in \mathfrak{A}$ is the unique element in the $W_{\text {aff-orbit of }} F\left(\lambda_{1} / \ell\right)+$ $w F\left(\lambda_{2} / \ell\right)$ (see section 2.2). Clearly,

$$
F^{-1}\left(\tilde{w}\left(F\left(\lambda_{1} / \ell\right)+w F\left(\lambda_{2} / \ell\right)\right)\right)=\tilde{w}\left(\lambda_{1} / \ell+w \lambda_{2} / \ell\right) .
$$

Moreover, $\ell\left(\tilde{w}\left(\lambda_{1} / \ell+w \lambda_{2} / \ell\right)\right)$ is in $P_{\ell}(G)$ by construction and is of the form $\tilde{w}^{\prime}\left(\lambda_{1}+w \lambda_{2}\right)$ for some $\tilde{w}^{\prime} \in W_{\text {aff }}$ such that $\tilde{w}^{\prime} \equiv \tilde{w}(\bmod \ell \check{Q})$. We denote this element in $P_{\ell}(G)$ (uniquely determined by the triple $\lambda_{1}, \lambda_{2}$ and $w$ ) by ${\overline{\lambda_{1}+w \lambda_{2}}}^{F}$.

Theorem 3.3 ([3], Proposition 3.5). With notation as before,

$$
M_{0}^{\mathrm{SL}(r)}(\underline{p}, \underline{\lambda}) \neq \emptyset \text { if and only if } n_{0}(\underline{\lambda}) \neq 0 .
$$

Theorem 3.4 (PRV for fusion product, 4]). Let $V_{\lambda_{1}}$ and $V_{\lambda_{2}}$ be finite dimensional irreducible $\mathfrak{s l}(r)$-modules with highest weights $\lambda_{1}$ and $\lambda_{2}$ respectively, with $\lambda_{1}, \lambda_{2} \in$ $P_{\ell}(\mathrm{SL}(r))$. Then, for any $w \in W_{\text {aff }}$, the irreducible $\mathfrak{s l}(r)$-module $V_{\overline{\lambda_{1}+w \lambda_{2}}} F$ occurs with multiplicity at least one in $V_{\lambda_{1}} \otimes^{F} V_{\lambda_{2}}$.

Proof. (P. Belkale informed me that he learned of the following derivation of PRV from Proposition 3.5 in 3 from C. Woodward.) Let $\mu_{i}=F\left(\lambda_{i} / \ell\right)$ for $i=1,2$, and $\mu_{3}=\tilde{w} F\left(\lambda_{1} / \ell+w \lambda_{2} / \ell\right)=\tilde{w}\left(\mu_{1}+w \mu_{2}\right)$, where $\tilde{w}\left(\mu_{1}+w \mu_{2}\right) \in \mathfrak{A}$ is the unique ele-

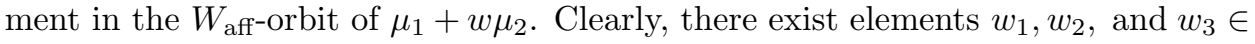
$W_{\text {aff }}$ such that $w_{1} \mu_{1}+w_{2} \mu_{2}+w_{3} \mu_{3}^{*}=0$, where $\mu_{3}^{*}$ denotes the dual of $\mu_{3}$ as in section 2.1. Thus, under the identification (*) of section $2.4, M_{0}^{\mathrm{SL}(r)}\left(\underline{p}, \lambda_{1}, \lambda_{2},\left({\overline{\lambda_{1}+w \lambda_{2}}}^{F}\right)^{*}\right)$ $\neq \emptyset$. Then it follows from Theorem 3.3 that $n_{0}\left(\lambda_{1}, \lambda_{2},\left({\overline{\lambda_{1}+w \lambda_{2}}}^{F}\right)^{*}\right) \neq 0$; equivalently $V_{{\overline{\lambda_{1}+w \lambda_{2}}}^{F}} \subset V_{\lambda_{1}} \otimes^{F} V_{\lambda_{2}}$ by the definition of the fusion product.

\section{Proof of Theorem 1.1}

In this section we restrict ourselves to $G=\mathrm{SL}(r)$. Recall that under the identification $(* *)$ of section 2.5 ,

$$
\operatorname{dim} H^{0}\left(M_{g}^{\mathrm{SL}(r)}(\underline{p}, \underline{\lambda}), \mathcal{L}(\underline{\lambda}, \ell)\right)=\operatorname{dim} V_{\mathcal{C}_{g}}^{\mathrm{SL}(r)}(\underline{p}, \underline{\lambda})=n_{g}(\underline{\lambda}) .
$$

Lemma 4.1. It suffices to show that Theorem 1.1 holds for $g=1$.

Proof. Using the 'factorization rule', Proposition $3.2(b)$, for any collection of weights $\underline{\lambda}$ in $P_{\ell}(\mathrm{SL}(r))$,

$$
\begin{aligned}
n_{g}(\underline{\lambda}) & =\sum_{\nu \in P_{\ell}(\mathrm{SL}(r))} n_{g-1}\left(\underline{\lambda}, \nu, \nu^{*}\right) \\
& =n_{g-1}(\underline{\lambda}, 0,0)+\sum_{\nu \in P_{\ell}(\operatorname{SL}(r)), \nu \neq 0} n_{g-1}\left(\underline{\lambda}, \nu, \nu^{*}\right) .
\end{aligned}
$$

By 'propagation of vacua', Proposition $\underline{3.2}(a), n_{g-1}(\underline{\lambda}, 0,0)=n_{g-1}(\underline{\lambda})$. Hence,

$$
n_{g}(\underline{\lambda}) \geq n_{g-1}(\underline{\lambda}) \text {. }
$$

Now suppose $n_{1}(\underline{\lambda})>0$. Clearly, by inequality $(2), n_{g}(\underline{\lambda})>0$ for any $g \geq 1$. Moreover, since $\nu+\nu^{*}$ is in the root lattice for any $\nu \in P$, we get the lower bound as claimed in Theorem 1.1 (under identification (1)) inductively by Proposition $3.2(b)$. 
Proposition 4.2. Theorem 1.1 holds for the case of one marking on $\mathcal{C}_{1}$ (i.e. for the particular case $g=1$ and $s=1$ ).

Proof. We will show that for any $\lambda \in P_{\ell}(\mathrm{SL}(r))$ that is also in the root lattice, there exists a special PRV-weight $\mu \in P_{\ell}(\mathrm{SL}(r))$ such that $V_{\mu} \subset V_{\lambda} \otimes^{F} V_{\mu}$. This will imply by the 'factorization rule' that $n_{1}(\lambda)>0$.

Given $\lambda=\sum_{i=1}^{r-1} a_{i} \omega_{i}$ that lies in the root lattice, by change of basis $\lambda=$ $\sum_{i=1}^{r-1} n_{i} \alpha_{i}$ for some $n_{i} \in \mathbb{Z}$. For any $w \in W_{\text {aff }}$, consider the equation

$$
\lambda=\mu^{\prime}-w \mu^{\prime} \text {. }
$$

There exists a $\mu^{\prime} \in P$ satisfying (3) if and only if $\lambda$ lies in the root lattice. The latter granted, we choose $w=w_{\alpha_{r-1}} w_{\alpha_{r-2}} \cdots w_{\alpha_{2}} w_{\alpha_{1}} \in W$. It is trivial to see that, for this particular choice of $w, \mu^{\prime}=\sum_{i=1}^{r-1}\left(n_{i}-n_{i-1}\right) \omega_{i}$ (with $n_{0}=0$ ) is the solution in $P$. Let $\tilde{w} \in W_{\text {aff }}$ such that $\mu:=\tilde{w}^{-1} \mu^{\prime}$ is in $P_{\ell}(\operatorname{SL}(r))$. Then we have

$$
\lambda=\tilde{w} \mu-w \tilde{w} \mu
$$

that is, $\overline{\lambda+w \tilde{w}}^{F}=\mu$. Thus, by Theorem 3.4, $V_{\mu} \subset V_{\lambda} \otimes^{F} V_{\mu}$; equivalently $n_{0}\left(\lambda, \mu, \mu^{*}\right)>0$. Then it follows from Proposition $\left[3.2(b)\right.$ that $n_{1}(\lambda)>0$. Hence the claim follows by equality (1).

Proposition 4.3. Theorem 1.1 holds for an arbitrary number of markings on $\mathcal{C}_{1}$.

Proof. We will show that $n_{1}(\underline{\lambda})>0$ whenever $\sum_{i=1}^{s} \lambda_{i}$ lies in the root lattice of $\mathrm{SL}(r)$.

Recall that for any $\lambda_{1}, \lambda_{2} \in P_{\ell}(\operatorname{SL}(r))$ and $w \in W_{\text {aff }}$, there exists $\tilde{w}^{\prime} \in W_{\text {aff }}$ such that $\tilde{w}^{\prime}\left(\lambda_{1}+w \lambda_{2}\right)$ is in $P_{\ell}(\operatorname{SL}(r))$. Moreover, by Theorem 3.4 the $\mathfrak{s l}(r)$ representation with highest weight $\tilde{w}^{\prime}\left(\lambda_{1}+w \lambda_{2}\right)$ appears in $V_{\lambda_{1}} \otimes^{F} V_{\lambda_{2}}$. Clearly, $\tilde{w}^{\prime}\left(\lambda_{1}+w \lambda_{2}\right)=\lambda_{1}+\lambda_{2}+\left(\tilde{w}^{\prime} \lambda_{1}-\lambda_{1}\right)+\left(\tilde{w}^{\prime} w \lambda_{2}-\lambda_{2}\right)$. Thus, if $\lambda_{1}+\lambda_{2}$ is in the root lattice, so is $\tilde{w}^{\prime}\left(\lambda_{1}+w \lambda_{2}\right)$ (see section 2.1).

We now repeat the above construction. Given weights $\lambda_{1}, \ldots, \lambda_{s} \in P_{\ell}(\operatorname{SL}(r))$ with $\sum_{i=1}^{s} \lambda_{i}$ in the root lattice, and $w_{1} \in W_{\text {aff }}$, there exists $w_{i}$ in $W_{\text {aff }}$ (which we find inductively) for $2 \leq i \leq s$ such that $w_{i}\left(\lambda_{i}+w_{i-1}\left(\lambda_{i-1}+\cdots+w_{2}\left(\lambda_{2}+w_{1} \lambda_{1}\right)\right)\right)$ is in $P_{\ell}(\mathrm{SL}(r))$. The last weight,

$$
\tilde{\lambda}:=w_{s}\left(\lambda_{s}+w_{s-1}\left(\lambda_{s-1}+w_{s-2}\left(\lambda_{s-2}+\cdots+w_{2}\left(\lambda_{2}+w_{1} \lambda_{1}\right)\right)\right)\right)
$$

is also in the root lattice. Moreover, by a repeated application of Theorem 3.4, we have $V_{\lambda_{1}} \otimes^{F} V_{\lambda_{2}} \otimes^{F} \cdots \otimes \otimes^{F} V_{\lambda_{s}} \supset V_{\tilde{\lambda}}$.

Since $V_{\lambda_{1}} \otimes^{F} V_{\lambda_{2}} \otimes^{F} \cdots \otimes \otimes^{F} V_{\lambda_{s}}$ decomposes with positive structure coefficients and the fusion product is associative, for any $\nu \in P_{\ell}(\mathrm{SL}(r))$

$$
\left(V_{\lambda_{1}} \otimes^{F} \cdots \otimes^{F} V_{\lambda_{s}}\right) \otimes^{F} V_{\nu}=V_{\lambda_{1}} \otimes^{F} \cdots \otimes^{F} V_{\lambda_{s}} \otimes^{F} V_{\nu} \supset V_{\tilde{\lambda}} \otimes^{F} V_{\nu} .
$$

In particular,

$$
n_{0}\left(\underline{\lambda}, \nu, \nu^{*}\right) \geq n_{0}\left(\tilde{\lambda}, \nu, \nu^{*}\right)
$$

where $\underline{\lambda}=\left(\lambda_{1}, \ldots, \lambda_{s}\right)$.

Using Proposition $\underline{3.2}(b)$,

$$
n_{1}(\underline{\lambda})=\sum_{\nu \in P_{\ell}(\operatorname{SL}(r))} n_{0}\left(\underline{\lambda}, \nu, \nu^{*}\right) \geq \sum_{\nu \in P_{\ell}(\operatorname{SL}(r))} n_{0}\left(\tilde{\lambda}, \nu, \nu^{*}\right),
$$


where the inequality in (5) holds by (4) term by term. Using Proposition $3.2(b)$ one more time, we get

$$
\sum_{\nu \in P_{\ell}(\operatorname{SL}(r))} n_{0}\left(\tilde{\lambda}, \nu, \nu^{*}\right)=n_{1}(\tilde{\lambda}) .
$$

By construction $\tilde{\lambda}$ is in root lattice and in $P_{\ell}(\mathrm{SL}(r))$. Thus, by Proposition 4.2, $n_{1}(\tilde{\lambda})>0$. Then it follows from $(5)$ and $(6)$ that $n_{1}(\underline{\lambda})>0$. Hence the claim, under the identification (1).

By the virtue of Lemma 4.1 and Proposition 4.3. Theorem 1.1 holds.

\section{ACKNOWLEDGMENTS}

This work is in response to a question of $\mathrm{P}$. Belkale, which asked if the dimension of $H^{0}\left(M_{g}(\underline{p}, \underline{\lambda}), \mathcal{L}(\underline{\lambda}, \ell)\right)$ is nonzero for $g \geq 1$. I am very thankful to him for many useful discussions and suggestions and for reading several earlier versions of this manuscript. I also thank S. Kumar, M.S. Narasimhan and T.R. Ramadas.

\section{REFERENCES}

[1] A. Beauville. Conformal blocks, fusion rules and the Verlinde formula. Proceedings of the Hirzebruch 65 Conference on Algebraic Geometry, Israel Math. Conf. Proc. (1996) 9: 75-96. MR 1360497 (97f:17025)

[2] A. Beauville and Y. Laszlo. Conformal blocks and generalized theta functions. Commun. Math. Phys. (1994) 164: 385-419. MR1289330 (95k:14011)

[3] P. Belkale. Quantum generalization of the Horn conjecture. J. Amer. Math. Soc., posted on October 25, 2007, PII: S 0894-0347(07)00584-X (to appear in print).

[4] P. Belkale. Private communication.

[5] U. Bhosle and A. Ramanathan. Moduli of parabolic G-bundles on curves. Math. Z. (1989) 202: 161-180. MR1013082 (90h:14018)

[6] N. Bourbaki. Groupes et Algèbres de Lie, Chaps. 4-6. Masson, Paris. 1981. MR647314 (83g:17001)

[7] J.-M. Drezet and M. S. Narasimhan. Groupe de Picard des variétés de modules de fibrés semi-stables sur les courbes algébriques. Invent. Math. (1989) 97: 53-94. MR 999313 (90d:14008)

[8] G. Faltings. A proof for the Verlinde formula. J. Alg. Geom. (1994) 3: 347-374. MR1257326 (95j:14013)

[9] G. Faltings. Stable G-bundles and projective connections. J. Alg. Geom. (1993) 2: 507-568. MR.1211997 (94i:14015)

[10] W. Fulton. Eigenvalues, invariant factors, highest weights, and Schubert calculus. Bull. Amer. Math. Soc. (N.S.) (2000) 37: 209-249. MR1754641 (2001g:15023)

[11] M. Gotô. A theorem on compact semi-simple groups. J. Math. Soc. Japan (1949) 1 (3): 270-272. MR0033829(11:497d)

[12] V. Kac. Infinite Dimensional Lie Algebras. Cambridge University Press, 1990. MR1104219 (92k:17038)

[13] A. Knutson and T. Tao. Honeycombs and sums of Hermitian matrices. Notices Amer. Math. Soc. (2001) 48 (2): 175-186. MR1811121 (2002g:15020)

[14] S. Kumar. Proof of the Parthasarathy-Rango Rao-Varadarajan conjecture. Invent. Math. (1988) 93: 117-130. MR943925 (89j:17009)

[15] S. Kumar, M. S. Narasimhan and A. Ramanathan. Infinite Grassmannians and moduli spaces of $G$-bundles. Math. Ann. (1994) 300: 41-75. MR.1289830 (96e:14011)

[16] Y. Laszlo and C. Sorger. The line bundles on the moduli of parabolic $G$-bundles over curves and their sections. Ann. Scient. École Norm. Sup. (1997) 30: 499-525. MR1456243 (98f:14007)

[17] O. Mathieu. Construction d'un groupe de Kac-Moody et applications. Compositio Math. (1989) 69 (1): 37-60. MR986812 (90f:17012) 
[18] V. B. Mehta and C. S. Seshadri. Moduli of vector bundles on curves with parabolic structures. Math. Ann. (1980) 248: 205-239. MR575939 (81i:14010)

[19] C. Pauly. Espaces de modules de fibrés paraboliques et blocs conformes. Duke Math. J. (1996) 84: 217-235. MR1394754 (97h:14022)

[20] C. Sorger. La formule de Verlinde. Séminare Bourbaki (1994), Astérisque (1996) 237: 87-114. MR:1423621 (98f:14009)

[21] C. Teleman and C. Woodward. Parabolic bundles, products of conjugacy classes, and Gromov-Witten invariants. Annales de l'Institut Fourier (Grenoble) (2003) 53 (3): 713748. MR2008438 (2004g:14053)

[22] A. Tsuchiya, K. Ueno and Y. Yamada. Conformal field theory on universal family of stable curves with gauge symmetries. Adv. Stud. Pure Math. (1989) 19: 459-566. MR 1048605 (92a:81191)

[23] E. Verlinde. Fusion rules and modular transformations in 2D conformal field theory. Nuclear Physics B (1988) 300: 360-376. MR954762 (89h:81238)

Université Paris 6, Case 7012, 2 Place Jussieu, 75251 Paris Cedex 05, France

E-mail address: boysal@math.jussieu.fr, arzu.boysal@boun.edu.tr 